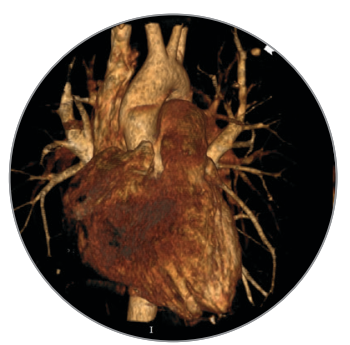

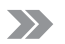

Palabras clave (DeCS)

Hemoptisis

Aneurisma

Tuberculosis

Arteria pulmonar

Radiología

\section{Key words (MeSH)}

Hemoptysis

Aneurysm

Tuberculosis

Pulmonary artery

Radiology

\title{
Pseudoaneurisma de Rasmussen: una rara, pero importante causa de hemoptisis. Presentación de un caso
}

\author{
Rasmussen's Pseudoaneurysm: A Rare But an Important Cause of \\ Hemoptysis. A Case Report
}

Camilo Grondona Ramírez Daniel Noreña Rengifo²

\begin{abstract}
Resumen
El pseudoaneurisma de Rasmussen es una de las causas de hemoptisis que a pesar de no ser muy común es importante conocer y tener en cuenta en el abordaje de estos pacientes. Típicamente ocurre en el contexto de la hemoptisis masiva y su abordaje, además de la estabilización del paciente, está enfocado en reconocerla como diagnóstico diferencial. El tratamiento inicial de estos pacientes incluye la radiografía de tórax convencional; sin embargo, la tomografía computarizada (TC) y la angioTC han mostrado el mayor rendimiento diagnóstico. Para su tratamiento, la embolización arterial transcatéter se ha consolidado como la técnica de elección; sin embargo, en algunos casos podría ser necesario el manejo quirúrgico abierto.
\end{abstract}

\section{Summary}

Rasmussen's pseudoaneurysm is one of the causes of hemoptysis that, despite not being very common, is important to recognize and take into account in the approach of these patients. It typically occurs in the context of massive hemoptysis and its approach in addition to patient stabilization is focused on recognizing it as a differential diagnosis. The initial approach for these patients includes conventional chest radiography; however, computed tomography (CT) and CTangiography have shown the highest diagnostic performance. For its treatment, transcatheter arterial embolization has established itself as the technique of choice; however, in some cases open surgical management may be necessary.

La hemoptisis, definida como el sangrado proveniente del tracto respiratorio inferior, es un escenario clínico que puede comprometer la vida y cuya etiología varía ampliamente. La mayoría de las enfermedades inflamatorias crónicas, incluyendo la tuberculosis, tienen el potencial de incrementar el flujo arterial sistémico pulmonar, lo que favorece la liberación de factores de crecimiento angiogenéticos y angioproteína 1 , los cuales a su vez promueven la neovascularización, remodelación vascular y el incremento en la formación de colaterales. Estas nuevas colaterales tienen la característica de ser vasos frágiles, con tendencia a la ruptura, lo que explica el aumento en el riesgo de hemoptisis en este grupo de pacientes. Sin embargo, a pesar de que el pseudoaneurisma de Rasmussen no es la principal causa de hemoptisis en los pacientes con tuberculosis, debe ser excluida dentro del abordaje diagnóstico para brindar un tratamiento oportuno y adecuado, que reduzca la morbimortalidad $(1,2)$.

El pseudoaneurisma de Rasmussen hace parte fisiopatológicamente de la infección posprimaria de la tuberculosis, caracterizada por la formación de una dilatación de la arteria pulmonar en una localización contigua o dentro de una caverna tuberculosa. Dicha dilatación es secundaria al debilitamiento progresivo de las capas media y adventicia de la arteria, que se inicia con el reemplazo de las mismas por tejido de granulación y posteriormente por fibrina, generando engrosamiento y la formación del mismo (3).

A continuación, se presenta un caso de hemoptisis secundaria a esta etiología, enfocado en su diagnóstico imagenológico.

\section{Presentación del caso}

Paciente masculino de 22 años de edad, con antecedente de reclusión en prisión durante 8 meses en 2016, quien después presentaba sudoración nocturna, astenia, adinamia, pérdida de peso y fiebre subjetiva. Consultó en enero de 2019 por cuadro clínico de tos seca y hemoptisis a repetición, con diagnóstico final de tuberculosis pulmonar con prueba cutánea de tuberculina de $10 \mathrm{~mm}$ y baciloscopias en las que se aislaron bacilos ácido-alcohol resistentes (BAAR). Se le inició tratamiento tetraconjugado (HRZE) y piridoxina.

Con el tratamiento tuvo mejoría parcial, pero recidivó a los 5 días; se presentó al servicio de urgencias con hematemesis progresiva con producción de coágulos y persistencia de los síntomas previos; además refería 
melenas y sensación de sed. Al examen físico se encontró en aceptables condiciones, taquicárdico, sin taquipnea, febril, con saturación de oxígeno adecuada y sin otros hallazgos anormales. Posteriormente se aclaró que la hematemesis referida por el paciente correspondía en realidad a hemoptisis. Los paraclínicos de ingreso reportaron leucocitosis con neutrofilia, prolongación del INR (International Normalized Ratio) y elevación de la PCR (Reacción en Cadena de la Polimerasa). Además, una radiografía de tórax extrainstitucional informaba cavitación del lóbulo superior derecho y adenopatías parahiliares.

Debido a la evolución tórpida, a pesar de la terapia, se le practicó la prueba de infección por VIH y nueva radiografía de tórax que mostró cavitación en el lóbulo superior derecho (figura 1), y tomografía computarizada (TC) de tórax con medio de contraste. Por el cuadro de aparente hematemesis se le realizó endoscopia digestiva superior la cual descartó hemorragia del tracto gastrointestinal. La TC de tórax demostró pseudoaneurisma de Rasmussen en el segmento apical del lóbulo superior izquierdo (figura 2). El paciente fue sometido a cirugía para el tratamiento del pseudoaneurisma.

\section{Discusión}

Hemoptisis es el término que se utiliza para definir el sangrado proveniente del tracto respiratorio inferior y que está relacionado con una gran variedad de etiologías, las cuales varían de acuerdo con la epidemiología local y el tipo de pacientes. En los pacientes ambulatorios las casusas más comunes de hemoptisis incluyen las infecciones respiratorias, la enfermedad pulmonar obstructiva crónica (EPOC), neoplasias malignas y bronquiectasias. En los países desarrollados, las bronquiectasias, las infecciones respiratorias y el cáncer de pulmón son las principales causas, mientras que en países en desarrollo, la tuberculosis y sus secuelas son la causa prevalente $(4,9)$.

La hemoptisis se ha definido de diversas maneras, según la cantidad de sangre y el efecto hemodinámico en el paciente. Actualmente, la guía del Colegio Americano de Radiología reconoce tres variantes de hemoptisis: masiva y que amenaza la vida, no masiva y sin amenaza de la vida y recurrente. La hemoptisis masiva y que amenaza la vida se define como aquella que pone al paciente en riesgo de asfixia o exanguinación y con un volumen mayor a $100 \mathrm{~cm}^{3}$ en 24 horas, con la hipotensión como factor independiente para definir esta categoría. En la variante masiva es importante definir si el paciente está estable o inestable. La variante no masiva y sin amenaza de la vida no cumple con los criterios de hemoptisis masiva. Finalmente, está la variante de hemoptisis recurrente, la cual es una nueva clasificación definida como episodios repetidos de hemoptisis, después de un tratamiento inicial con terapia médica o embolización, que usualmente no amenazan la vida y tienen una causa conocida.

En Colombia, la tuberculosis tiene una incidencia de 17,7 casos por 100.000 habitantes al 2018, según datos del Boletín Epidemiológico Semanal del Instituto Nacional de Salud y MinSalud (6).

El pseudoaneurisma de Rasmussen es un fenómeno raro, con una prevalencia de $5 \%$ en pacientes con tuberculosis crónica cavitaria, que se presenta en la etapa posprimaria de la infección tuberculosa; se define como una dilatación de la arteria pulmonar en un sitio contiguo o incluso dentro de una caverna tuberculosa formada durante la infección primaria. Los mecanismos que preceden dicha dilatación están dados por el depósito de tejido de granulación y posterior depósito de fibrina en las capas media y adventicia de la arteria, lo que se traduce en engrosamiento de la misma, debilitamiento de la pared arterial y producción de un pseudoaneurisma, que se puede romper (3).

En el abordaje diagnóstico imagenológico de la hemoptisis se han definido estrategias por parte del Colegio Americano de Radiología que van a variar en relación con el tipo de presentación clínica. En los pacientes con hemoptisis masiva, la broncoscopia para taponamiento y limpieza de coágulos es el pilar inicial de manejo, especialmente en el subgrupo de pacientes con inestabilidad hemodinámica. Adicionalmente, se recomienda la radiografía de tórax para evaluar la extensión del compromiso pulmonar y la localización del tubo endotraqueal. Otros métodos incluyen la angiografía con embolización, cuyo objetivo es terapéutico y no diagnóstico, con tasas de éxito entre $70 \%$ hasta $99 \%$ de acuerdo con la serie. En este escenario, tanto la tomografía con medio de contraste como la angiotomografía tienen un nivel de evidencia alto para el diagnóstico y la localización de la fuente de sangrado, razón por la cual están recomendadas. En hemoptisis no masiva, la tomografía con medio de contraste es el método inicial para determinar la etiología, limitando la tomografía sin contraste para aquellos pacientes con contraindicaciones (pobre función renal o alergia al medio de contraste). El uso de la angiotomografía en este grupo de pacientes también se recomienda, en especial cuando se está realizando la planeación de la embolización angiográfica, la cual, a pesar de no ser de primera línea, ha mostrado un incremento en su uso con el paso del tiempo. Finalmente, la radiografía de tórax continúa siendo una elección razonable como estudio de imagen inicial, especialmente cuando se desea confirmar un diagnóstico clínico benigno (neumonía). Por último, la hemoptisis recurrente tendrá como pilares de su evaluación la radiografía de tórax, la tomografía con medio de contraste y la angiotomografía con unas consideraciones similares a las descritas previamente (7).

En cuanto al diagnóstico particular del pseudoaneurisma de Rasmussen, la radiografía de tórax es un buen método inicial que permite revelar el sitio de sangrado en aproximadamente $46 \%$ de los casos y la causa en un $35 \%$ de los casos, aproximadamente. Sin embargo, la TC con medio de contraste y la angioTC se han convertido en las modalidades iniciales de estudio de este grupo de pacientes por su precisión y disponibilidad.

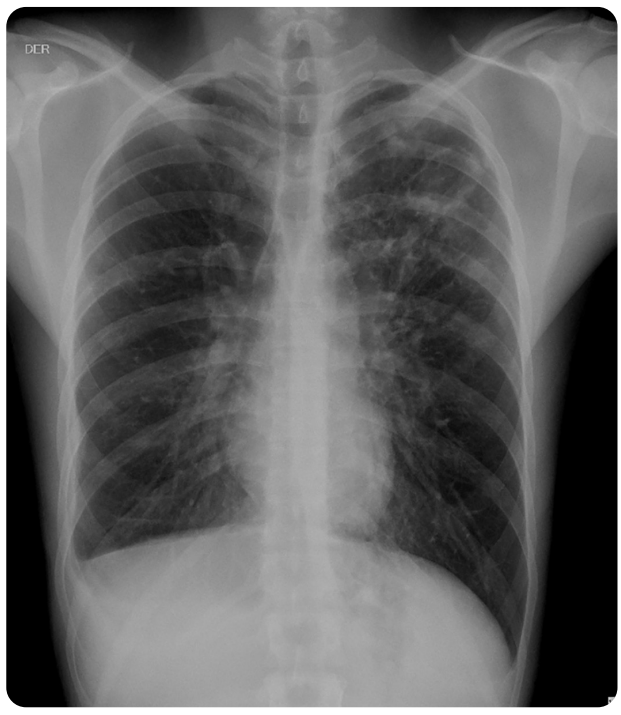

Figura 1. Radiografía de tórax en proyección posteroanterior. Cavitación en el lóbulo superior izquierdo y opacidades retículonodulares bilaterales de predominio en lóbulos superiores. 

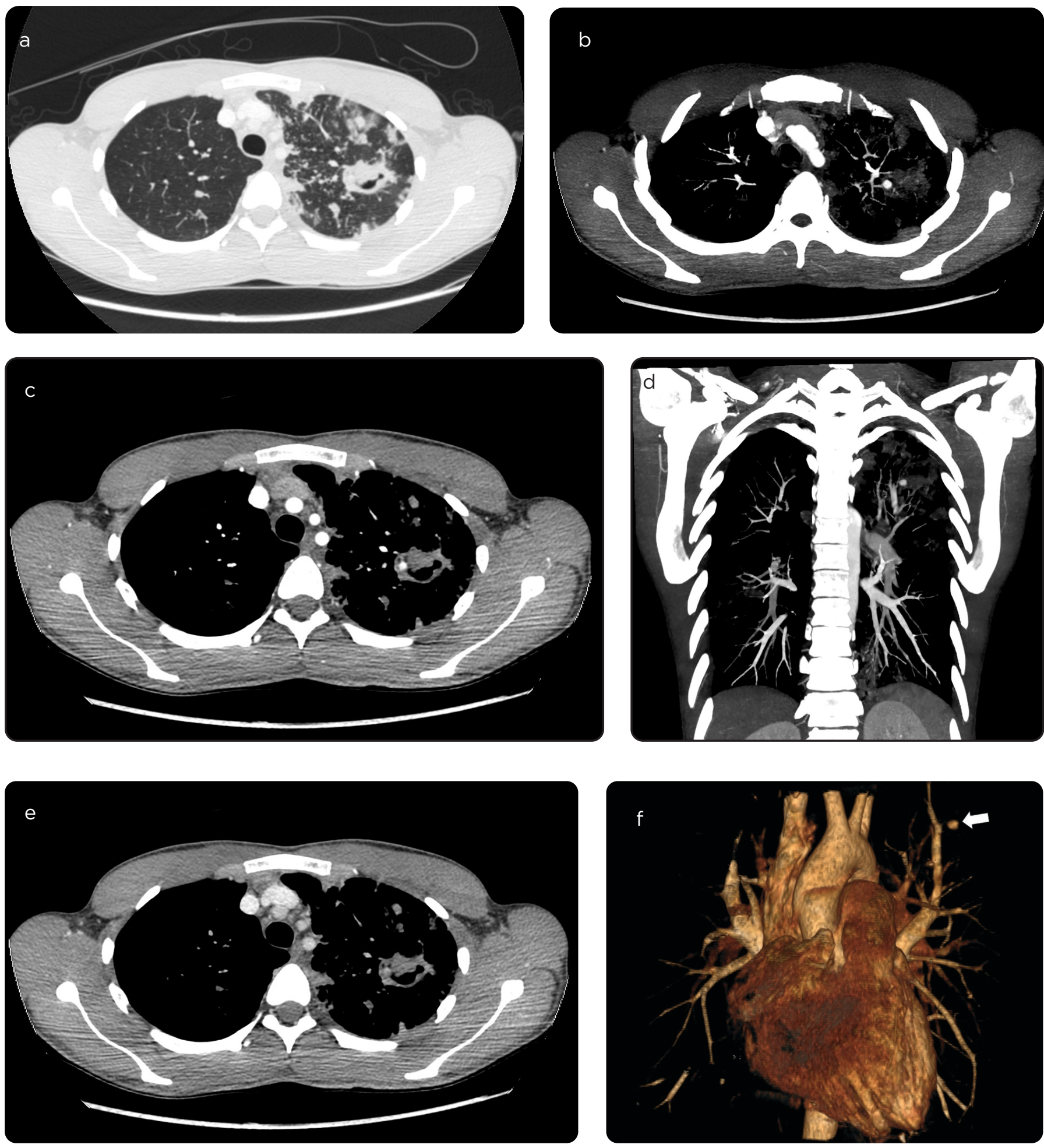

Figura 2. Tomografía de tórax con medio de contraste. a) cavitación en el lóbulo superior izquierdo, nódulos y micronódulos que comprometen principalmente los lóbulos superiores y en especial el izquierdo, evidentes en el corte axial en ventana pulmonar. b) En la fase arterial lesión nodular con realce prominente dentro de la cavitación, que se comporta igual a las arterias pulmonares tanto en la fase arterial como en c) en la fase venosa. d y e). En el corte axial y la reconstrucción coronal con proyección de máxima intensidad (MIP) se demuestra que la lesión depende de rama para el segmento ápico-posterior izquierdo. f) Reconstrucción tridimensional, demuestra la localización del pseudoaneurisma (flecha blanca). 
El pseudoaneurisma se identificará como un nódulo que realza ávidamente en la fase arterial, con lavado en la fase venosa, pero que conserva su morfología y se localiza dentro o adyacente a una cavitación tuberculosa $(1,13,16)$.

El tratamiento de elección es la embolización arterial transcatéter precoz por sus altas tasas de éxito y con múltiples elementos comerciales disponibles para la embolización, como las embosferas, los coils, adhesivos tisulares, esponjas de gelatina, los balones y stents; los cuales reemplazan en la mayoría de los casos la cirugía abierta $(16,17)$.

El tratamiento conservador tiene muy alta mortalidad en los casos en que se presenta con hemoptisis masiva y puede ir del 50 al $100 \%$ de los pacientes. En algunas ocasiones se puede requerir manejo quirúrgico $(17,18)$.

El manejo quirúrgico fue la técnica de elección durante mucho tiempo para el control de la hemoptisis masiva y recurrente; sin embargo, tiene una alta tasa de mortalidad de aproximadamente el $20 \%$, que en comparación con la alta mortalidad del tratamiento conservador, justifican la intervención. La indicación para intervención quirúrgica se plantea ante pacientes con sangrado unilateral incontrolable que no responden a otras terapias de primera línea y cuya reserva pulmonar justifique el tratamiento. Dentro de las técnicas quirúrgicas se incluye la constricción quirúrgica (banding) de la arteria pulmonar o resección del aneurisma asociado a reemplazo protésico $(17,18)$.

El caso de este paciente es una excelente forma de demostrar cómo, a pesar de que el pseudoaneurisma de Rasmussen no es una de las principales causas de hemoptisis, en la población colombiana, por ser un país en desarrollo, siempre debe considerarse dentro del diagnóstico por la alta incidencia de tuberculosis. El manejo y los desenlaces van a ser, entonces, altamente dependientes del abordaje inicial, de acuerdo con el escenario clínico establecido, como en este caso, que permitió hacer un diagnóstico y tratamiento oportuno.

\section{Referencias}

1. Bruzzi JF, et al. Multi-detector row CT of hemoptysis. Radiographics. 2006;26:3-22.

2. Rajamannar KV, Kilaru H, Aravelly S, Gudipati AR, Kilaru SC. Massive hemoptysis from Rasmussen's aneurysm in active pulmonary tuberculosis; A case report of successful treatment with bronchial artery embolization. Respir. Med. Case Reports. 2017;22:277-9.

3. Kim HY, et al. Thoracic sequelae and complications of tuberculosis. RadioGraphics. 2001;21:839-58.

4. Jones R, Charlton J, Latinovic R, Gulliford MC. Alarm symptoms and identification of non-cancer diagnoses in primary care: Cohort study. BMJ. 2009;339:491-3.

5. Abdulmalak C, et al. Haemoptysis in adults: A 5-year study using the French nationwide hospital administrative database. Eur. Respir. J. 2015;46:503-11

6. Fartoukh M, et al. An integrated approach to diagnosis and management of severe haemoptysis in patients admitted to the intensive care unit: A case series from a referral centre. Respir. Res. 2007;8:1-9

7. Olsen KM, et al. ACR Appropriateness Criteria ${ }^{\circledR}$ Hemoptysis. J. Am. Coll. Radiol. 2020;17:S148-59.

8. Tom LM, et al. Recurrent bleeding, survival, and longitudinal pulmonary function following bronchial artery embolization for hemoptysis in a U.S. adult population. J. Vasc. Interv. Radiol. 2015;26:1806-13.e1.

9. Panda A, Bhalla AS, Goyal A. Bronchial artery embolization in hemoptysis: A systematic review. Diagnostic Interv. Radiol. 2017;23:307-17.

10. Sakr L, Dutau H. Massive hemoptysis: An update on the role of bronchoscopy in diagnosis and management. Respiration. 2010;80:38-58.

11. Uzun O, Atasoy Y, Findik S, Atici AG, Erkan L. A prospective evaluation of hemoptysis cases in a tertiary referral hospital. Clin. Respir. J. 2010;4:131-8.

12. Kervancioglu S, Bayram N, Yilmaz FG, Sanli M, Sirikci A. Radiological findings and outcomes of bronchial artery embolization in cryptogenic hemoptysis. J. Korean Med. Sci. 2015;30:591-7.

13. Peghini Gavilanes E, Fernández-Velilla M, Bermejo Moriñigo A, Morales Ruiz R. Pseudoaneurisma de Rasmussen: hallazgos en tomografía computada. Revisión de casos. Rev. Argentina Radiol. 2016;80:122-6.
14. Instituto Nacional de Salud. Una mirada hacia el comportamiento de la tuberculosis en Colombia, 2018 (Semanas epidemiológicas 1-36). 35 (2018).

15. Shih SY, Tsai IC, Chang YT, Tsan YT, Hu SY. Fatal haemoptysis caused by a ruptured Rasmussen's aneurysm. Thorax. 2011;66:553-4.

16. Neelakantan S, Anandarajan R, Swamy AK. Rare cause of massive haemoptysis in pulmonary tuberculosis: Rasmussen's aneurysm. BMJ Case Rep. 2016;3-5.

17. Ossés JM. ¿Qué sabemos de los aneurismas de la arteria pulmonar? Rev. Am. Med. Respir. 2014;14:92-3.

18. Vicente Antunes SI, Rodríguez Martín P, de San Pablo Sánchez AM. Hemoptisis Cirugía en Patología Pulmonar no tumoral. 2010;XVI:147-59.

\section{Correspondencia}

Brian Daniel Noreña Rengifo

Facultad de Medicina, Universidad de Antioquia

Calle 70 \# 52-21

Medellín, Colombia

Brian.norena@udea.edu.co

Recibido para evaluación: 10 de junio de 2020

Aceptado para publicación: 26 de agosto de 2020 